\title{
Automated Scientific Assistant for Cancer and Chemoprevention
}

\author{
Sotiris Lazarou, Antonis C. Kakas, Christiana Neophytou, \\ and Andreas Constantinou \\ Departments of Computer Science and Biological Sciences, \\ University of Cyprus, P.O. Box 20537 - 1678 Nicosia, Cyprus
}

\begin{abstract}
Logical modeling of cell biological phenomena has the potential to facilitate both the understanding of the mechanisms that underly the phenomena as well as the process of experimentation undertaken by the biologist. Starting from the general hypotheses that Scientific Modeling is inextricably linked to abductive reasoning, we aim to develop a general logical model of cell signalling and to provide an automated scientific assistant for the biologists to help them in thinking about their experimental results and the possible further investigation of the phenomena of interest. We present a first such system, called ApoCelSys, that provides an automated analysis of experimental results and support to a biology laboratory that is studying Cancer and Chemoprevention.
\end{abstract}

Keywords: Abductive Reasoning, Systems Biology, Cancer Modeling.

\section{Introduction: Scientific Modeling in Logic}

Modeling scientific phenomena requires that we set up a link between our mental understanding of the phenomena and the empirical information that we collect from observing the phenomena. In most cases the scientific effort concentrates on setting up experiments that would provide empirical information suitable for developing further (in the extreme case abandoning) our current model for the phenomena. An abstraction therefore is required both at the object level of understanding the processes that generate the phenomena and at the meta-level of understanding how to set up experiments that would reveal useful information for the further development of our scientific model. Using logic as the basic for scientific modeling facilitates this abstraction at both levels and the connection between them. For Molecular Biology, logic is particularly suited as (at least currently) the theoretical models of cell biology are more at the descriptive level and experiments are developed following a rationale at the qualitative level rather than the quantitative level.

The purpose of the work in this paper is two fold. To develop logical models of biological process through which we can carry out a biologically valid analysis of experimental (in-vivo or in-vitro) results and to provide based on this automated help to the Biologists in thinking about the scientific process of their experiments and their possible further investigation of the phenomena they are studying. The 
aim is to (incrementally) develop an underlying model for signal propagation in a cell that is independent of any particular biological phenomena and process on top of which we can then modularly build a model for specific biological problems. The underlying model needs to capture the propagation of increased as well as that of decreased activation, where the increase and decrease are determined through relative changes with respect to a normal level of activity.

We present a model for cell signaling formulated within the framework of Abductive Logic Programming (ALP), together with a tool to be used as an automated assistant by the biologist. The study is carried out with emphasis on the particular biological process of apoptosis as studied by the group of Cancer Biology and Chemoprevention at the Department of Biological Sciences at the University of Cyprus. Their aim is to investigate the molecular mechanisms through which natural substances or their derivatives participate in the inhibition of carcinogenesis (chemoprevention) or the inhibition of tumor progression.

\section{Biological Background}

Physiological processes are conducted in cells through sequences of biochemical reactions known as molecular pathways. Each molecular pathway consists of a series of biochemical reactions that are connected by their intermediates: the products of one reaction are the substrates for subsequent reactions, and so on. Apoptosis or programmed cell death (PCD) is a physiological process which is vital for normal development and elimination of damaged cells in living organisms (reviewed in [2]). Apoptosis is a main tumor suppressor mechanism within the body because it gets rid of cells that have extensive DNA damage. Its deregulation can lead to cancer and other diseases.

Chemotherapy primarily refers to the treatment of cancer with an antineoplastic drug. Evidence in the literature suggests that Vitamin E natural and synthetic analogues may target the main survival pathways of the cell (i.e., PI3K and NF-kB), which provides not only an amplification response of the apoptotic pathway, but also kills selectively cancer cells whose survival may depend on activating these pathways [16. The goal of our biological study is to investigate the molecular events by which a Vitamin E synthetic derivative (VitESD) leads to apoptosis in breast cancer. For this purpose, we use two breast cancer cell lines, MCF-7 and MDA-MB-231, which are widely used as tumor models.

In vitro experiments are time-consuming and expensive as we need to screen a large number of possible targets in order to identify proteins that are involved in the signaling cascade affected by the compound under investigation. The use of an automated system will allow for calculated suggestions of molecules likely to be involved in the apoptotic pathway induced by the drug.

\section{Scientific Modeling in Abductive Logic Programming}

Scientific modeling is inextricably linked to abductive reasoning. Abductive explanations for new observational data, that are based on the current form of 
our model, generate new information that helps to develop further the model. In effect, abductive reasoning rationalizes the empirical data with respect to the current model with further hypotheses on the incompleteness of the model. Abductive Logic Programming (ALP) 43] provides a framework for logical modeling that also directly supports abductive reasoning and hence it is particulary suited for scientific modeling. A model or a theory, $T$, in ALP is described in terms of a triple $(P, A, I C)$ consisting of a logic program, $P$, a set of abducible predicates, $A$, and a set of classical logic formulas $I C$, called the integrity constraints of the theory. The program $P$ contains definitional knowledge representing the general laws about our problem domain through a complete definition of a set of observable predicates in terms of each other, background predicates (which are again assumed to be completely specified in $P$ ) and a set of abducible predicates that are open and which thus carry the incompleteness of the model. The integrity constraints, $I C$, represent assertional knowledge of known properties of the domain that must necessarily be respected and maintained when we complete further our theory. Given such an ALP theory the abductive reasoning that infers an abductive explanation is defined as follows.

Definition 1. Given an abductive logic theory $(P, A, I C)$, an abductive explanation for an observation $O$, is a set, $\Delta$, of ground abducible atoms on the predicates $A$ such that:

$-P \cup \Delta=_{L P} O$

$-P \cup \Delta \models{ }_{L P} I C$.

where $\models_{L P}$ denotes the logical entailment relation in Logic Programming.

The abductive explanation $\Delta$ represents a hypothesis, which together with the model described in $P$ explains how the experimental observations, $Q$ could hold. The role of the integrity constraints $I C$ is to impose additional validity requirements on the hypotheses $\Delta$. They are modularly stated in the theory, separately from the basic model captured in P. As such, they can also be used, as we will see below, to steer the search for specific forms of explanation that our domain experts are looking for.

\section{Modeling Apoptosis and Drug Effects}

The core of our logical model consists of a fairly general representation of the activity of Signal Propagation in a cell. The task is that given a network of pathways generated through biological experiments, such as the one shown in Figure 1, to logically model the signal propagation over this and the effect of the presence of external drugs on this propagation.

We will consider the propagation of two types of signals: positive signal and negative signal. Normally cell signalling refers to the propagation of a positive signal, namely the iterative increase in activity of molecules along the pathway. In the propagation of a negative signal we refer to the iterative decrease in activity of molecules along the pathway. The increase and decrease of activity 
are taken relative to some level which is considered to be the normal background level of activity. The propagation of a negative signal is appropriate when we want to follow the inhibition effects of molecules. The core principles of positive and negative signal propagation are respectively the following.

Positive Propagation. The activation of a molecule $\mathrm{X}$ will cause the activation of a molecule $\mathrm{Y}$ that follows $\mathrm{X}$ (i.e. $\mathrm{Y}$ is induced by $\mathrm{X}$ ) in a signalling pathway, provided that $\mathrm{Y}$ or this reaction from $\mathrm{X}$ to $\mathrm{Y}$ is not inhibited by the activation of another molecule.

Negative Propagation. The inactivation of a molecule $\mathrm{X}$ will cause the inactivation of a molecule $\mathrm{Y}$ that follows $\mathrm{X}$ in a signalling pathway, provided there is no other molecule $\mathrm{Z}$ that also induces $\mathrm{Y}$ and $\mathrm{Z}$ is activated.

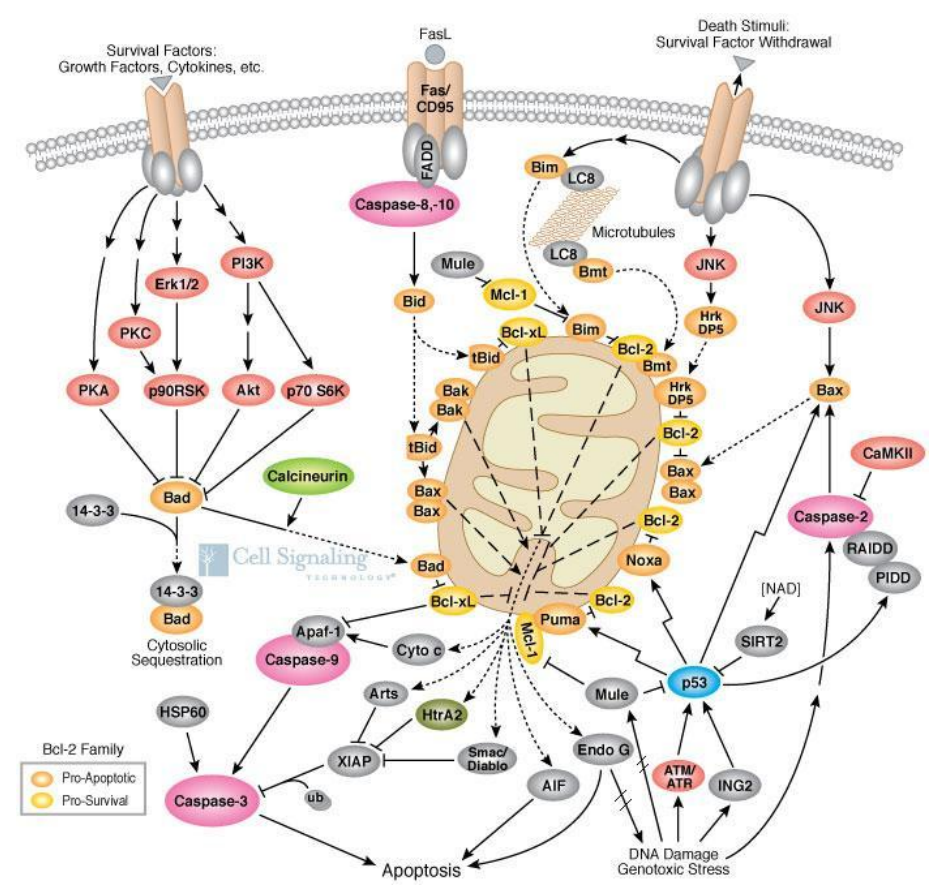

Fig. 1. Apoptosis pathways linked to the Mitochondrial

In these principles we abstract away the details of the actual chemical processes that occur to bring about a single step of propagation and we do not distinguish between the different types of signal propagation that are biologically possible. In general, this high-level abstract view of a single step of propagation is depicted in Figure 2 where the biological pathways are abstracted into a graph with positive or inducement links and negative or inhibitory links. Hence the substance $\mathrm{D}$ will be activated when any one of $\mathrm{A}$ and $\mathrm{B}$ are activated but 


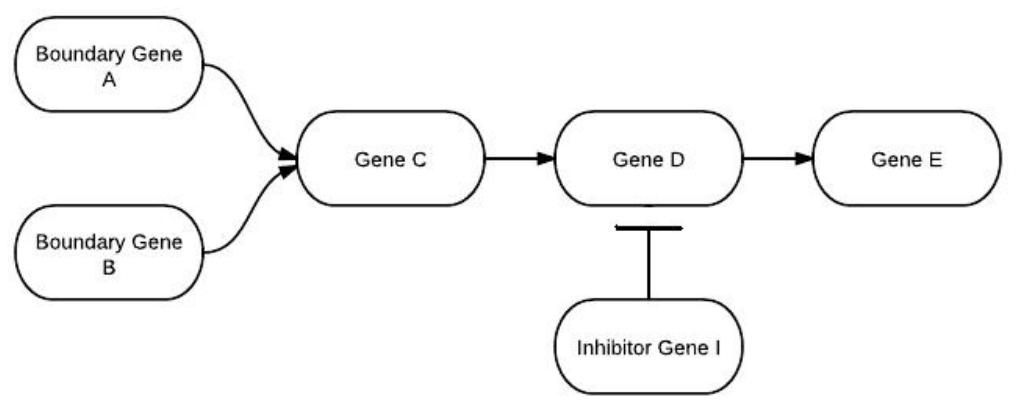

Fig. 2. An abstraction of Cell Signalling

its inhibitory gene, I, is not activated. Similarly, D will be inactive when $\mathrm{C}$ is inactive or when $I$ is activated.

Under the normal operation of the cell signals start from some boundary points, such as A or B. When an external drug is added in the cell then we have the possibility that this could activate or inactivate a molecule at any part of the network (or even enable new hitherto unknown links in the network) present in the background network. The task of the biological research in which the logical model and its automation is to assist, is to discover the effect of the drug on these background networks and the possible existence of other pathways relevant to the cell operation that is studied, e.g. apoptosis, as in the case of our study.

The above basic principles of signal propagation are captured by the following recursive rules for active/2 and inactive/2 in a logic program, $P$, together with the a set of integrity constraints, $I C$, of the theory $T=<P, A, I C>$ in ALP.

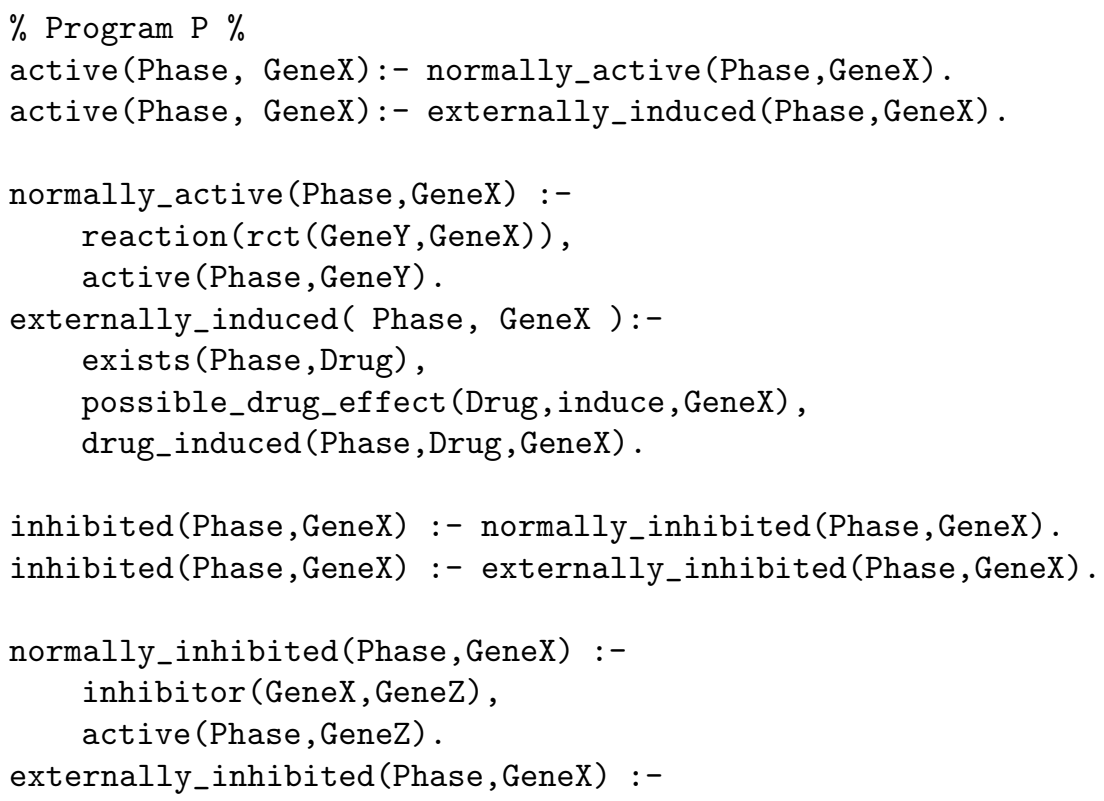




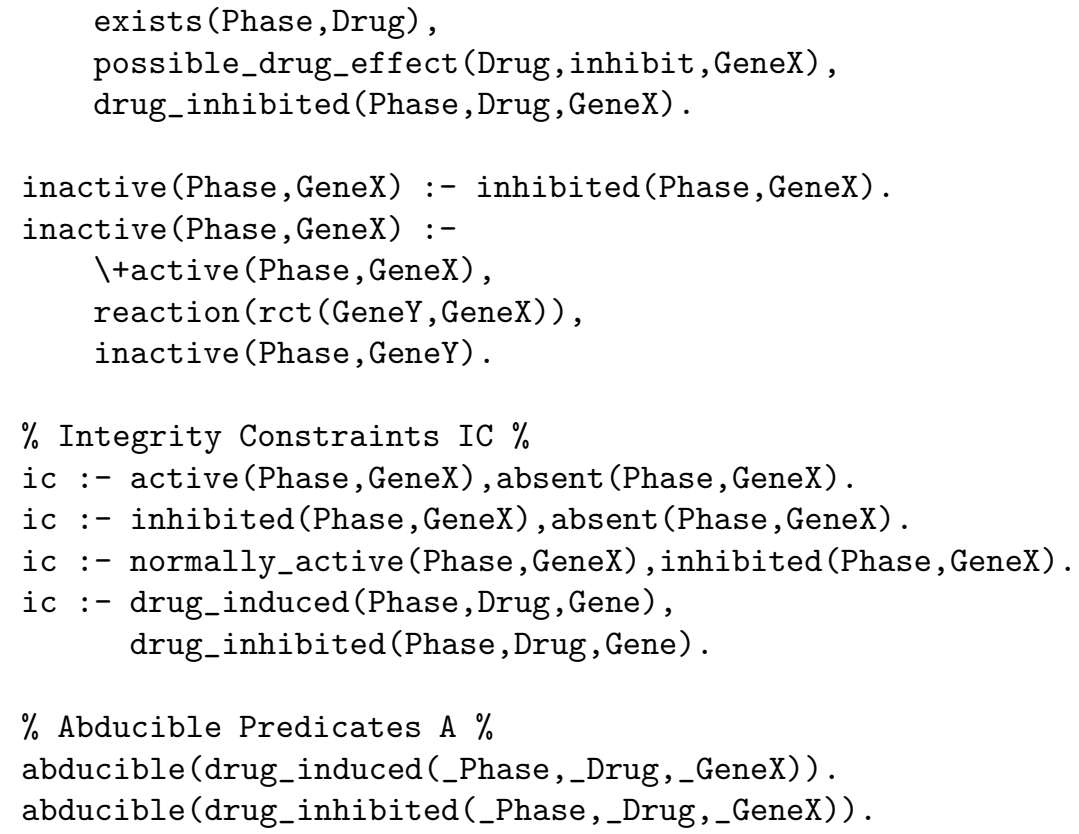

\section{$\%$ Integrity Constraints IC \%}

ic :- active (Phase, GeneX), absent (Phase, GeneX).

ic :- inhibited (Phase, GeneX), absent (Phase, GeneX).

ic :- normally_active (Phase, GeneX), inhibited (Phase, GeneX).

ic :- drug_induced (Phase, Drug, Gene), drug_inhibited (Phase, Drug, Gene).

\section{$\%$ Abducible Predicates A \%}

abducible (drug_induced(_Phase,_Drug,_GeneX)) .

abducible(drug_inhibited(_Phase,_Drug,_GeneX)) .

The "Phase" variable in the model relations distinguishes experiments where different drugs could be present with different possible effects of these drugs as captured by the lower-level predicate, possible_drug_effect/3, defined via some biological knowledge that we have a-priori of the drugs or via some hypothetical behavior of the drug to which the biologist wants to restrict their attention of study. The predicate absent $/ 2$ is also used to describe the variation in the set up of the various experiments where the biologist will arrange for some molecules to be effectively taken out the cell and hence to signal can go through them.

The reaction $/ 2$ and inhibitor $/ 2$ relations are given directly from the biological background pathway networks in the literature (see for example figure 1 again) as a database of extensional information of the following form:

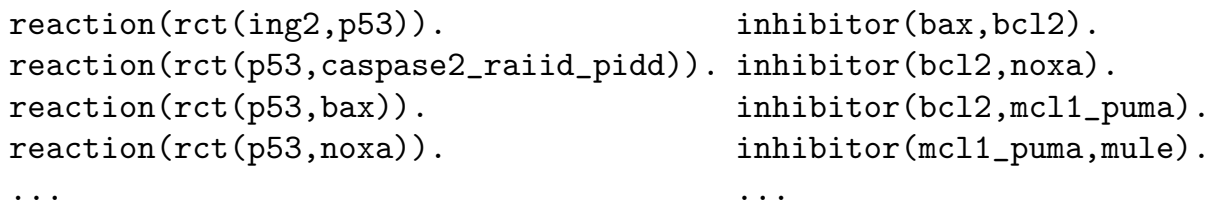

The observable predicates in the model are the two predicates of active/2 and inactive/2 through which the observations in any single experiment are turned into a conjunctive query for an abductive explanation through the model of $T$. Observations are of two types: activation or inactivation of some molecule from which it is known (or assumed in the model) that some overall operation of the cell, such as apoptosis will ensue or not and the activation or inactivation of intermediate molecules (markers) that the experimenter has measured. 
In the above model the only abducible predicates refer to assumptions that we can make on the effect of the Drugs on the molecules. Other abducible predicates can be added if we want to extend the model to cover the possibility of some other unknown external effect on the molecules or indeed the possibility of the existence of other unknown background reactions.

\section{ApoCelSys: A Scientific Advisor System}

ApoCelSys is a system that uses the model described in the previous sections to simulate the essential function of apoptosis, otherwise known as programmed cell death. The main purpose of the system is the creation of explanatory assumptions for validating the phenotype that was observed by the user and presenting a set of hypotheses on the effect of the synthetic substance introduced in the cell.

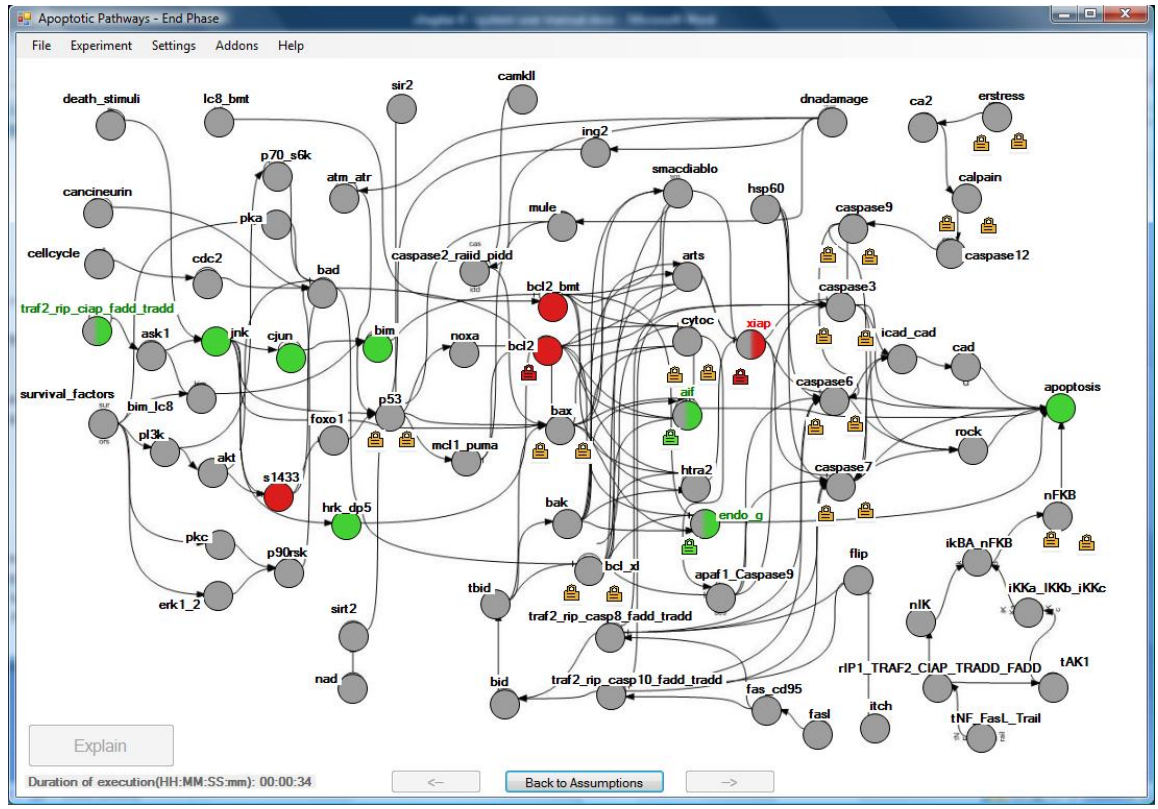

Fig. 3. System Interface of ApoCelSys and Propagation of an Explanation

A user interface, shown in figure 3 , provides a graphical representation of the molecular pathways leading to apoptosis. This helps to simplify the insertion of the experimental data and the observations to be explained, whether these come from real in-vitro or hypothetical experiments. The user can set the status of any gene to any of the five predefined statuses: "active" either "fixed" by the experimenter or "observed"; "inactive" similarly either "fixed/absent" or "observed" or "neutral" otherwise. Also the possible effect of the drug on the 
gene can be regulated by the user if and when such information is available. Otherwise, the system assumes that the drug can possibly affect the gene in either way of inducing or inhibiting the gene.

The user can save this experimental data so that s/he can collect and easily revisit a set of experiments that have been analyzed by the system. After passing the experiment's information as input and executing the query, the system displays the first explanation found using distinguishing colors on the corresponding elements (red for the assumption of drug inhibited and green for drug induced).

A tool for enumerating the explanations is also available through which the user can view, filter and present the most interesting, in her opinion, explanation for a more detailed examination. During this single explanation display, the system also allows the propagation of the substances' primary effect on the rest of the cell for an overview of the pathway activation and in particular the way that apoptosis is activated or not. An example of this can be seen in figure 3 where the explanation that the drug induces traf2_rip_ciap_fadd_tradd, aif, endo_g and inhibits $X I A P$ propagates to activate $j n k, c j u n, \ldots$ and this eventually propagates to activate apoptosis. It also shows how this explanation has an inactivation effect on $s 1433$ and $b c l 2 \_b m t$.

Frequency Charts for Multiple Explanations. Due to the multiplicity of the explanations that can be generated, automated analysis tools provide effective views on the likelihood of each assumption on the entire spectrum of explanations. The use of this tool creates graphs that exhibit the probability of the assumption of inducing or inhibiting elements throughout all produced explanations. An example is shown in figure 4 where we can see that aif and endo_g have the most hits in being induced by the synthetic substance and that as a second choice the most probable effects are the inducements of bak, xiap, tbid and bid. In addition, if requested, the system is in a position to compute, analyze and present charts displaying the likelihood of any gene being activated irrespective of being a direct drug effect or of being a consequence of the latter. This information can be interpreted as displaying all possible pathways along with their appearance frequency, thus enabling the user to better judge the most probable pathways to apoptosis. These visual tools allow the easy comparison by the user between multiple variations of an experiment or even between cross-experiments.

\section{Validation and Evaluation of the System}

In order to validate the system we have analyzed the experimental results of real in-vitro experiments as described in section 2 for investigating the action of $V i t E S D$. One such experiment was carried out with the MCF-7 cancel cellline and another one with the MDA-MB-231 cell-line. The purpose was to judge the appropriateness of the hypotheses produced but also to ascertain the help provided to the biologists in: (1) analyzing the results of their experiments and (2) allowing them to develop further their investigation by carrying out thought experiments that they deemed useful. 


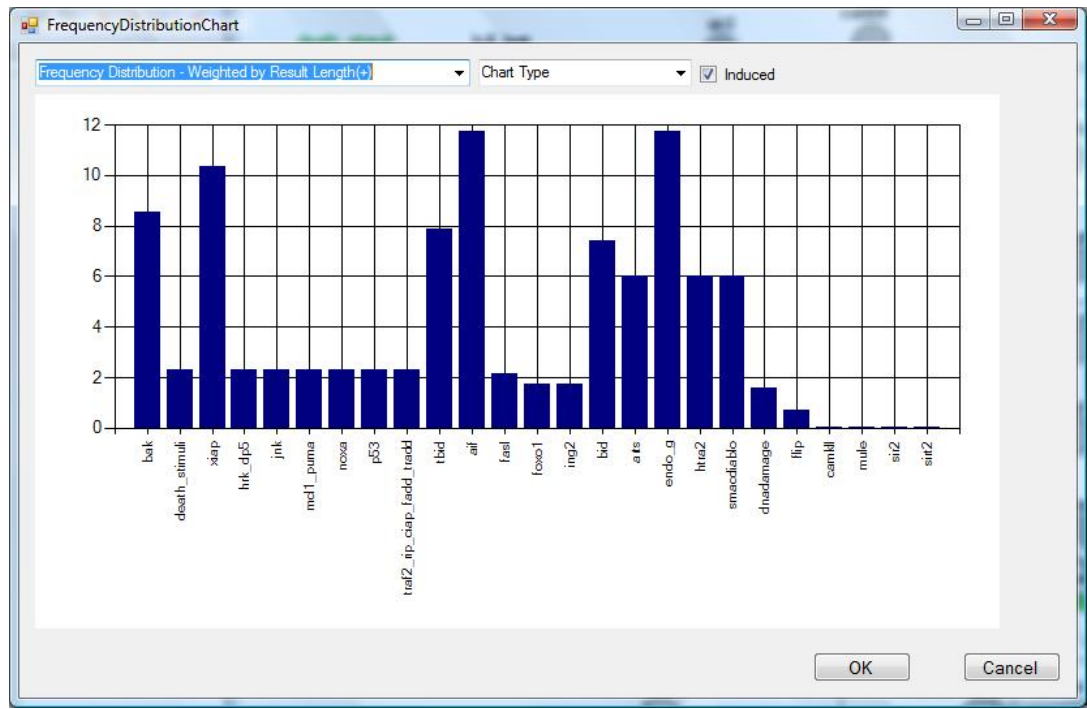

Fig. 4. Frequency Distribution across Explanations

These two experiments were analyzed with the ApoCelSys system and then the results were given back to the biologist to evaluate them. For the MCF7 experiment the results suggest that Bak and tbid are greatly induced and that Smac/Diablo is also moderately activated by the presence of $V i t E S D$. The ApoCelSys system therefore suggests that most likely $V i t E S D$ helps to activate apoptosis through the intrinsic pathway of apoptosis. The frequency distribution chart, generated by the ApoCelSys system, showing molecules that become inactive in the presence of the drug, suggests that PI3K, the upstream regulator of AKT, is in fact inhibited during apoptosis in our system and that Calcium levels (Ca2)-which control Calpain and subsequently Caspase activation-are also diminished. These findings are in line with the central role that AKT may play and further attenuate the caspase-independent mode of action of the VitESD.

Similarly, interesting results were produced for the second experiment with the MDA-MB-231 cells. Furthermore, in both cell lines, Survival factors such as growth proteins are suggested to become inactivated in the presence of the drug. This highlights another unexplored possibility that could explain the induction of apoptosis in our system: the inhibition of Growth Receptors on the cell membrane. The implication of these receptors coincides with the inactivation of PI3K and AKT which as mentioned before seem to play a central role in our system.

\subsection{Expanding the Biological Investigation}

The development of scientific theories often requires broadening the domain of investigation in several ways. For example, it may be relevant to combine the study of the cell process we are interested in with that of other cell processes 
that we know or suspect that are related to our primary interest. During the process of developing the ApoCelSys system the biologists expanded their investigation to the proliferation process of the cancer cells in addition to that of apoptosis. This required that the underlying pathways network to contain also the main established pathways for cell proliferation, some of which have cross over points with the apoptosis networks. The modular and logical nature of our model allowed this to be carried out in a straightforward and immediate way.

In-silico Thought Experiments. Scientists often use thought experiments for guiding them in their future investigations. These can help in understanding the significance of new ideas and how they can help to expand the horizon of investigation. The ApoCelSys system facilitates the examination of experiments that the Biologist may want to hypothetically ask. For example, what would the explanatory effect of the drug be if we also observed some other molecules in the cell? How would the experimental results be explained if we added new information in the model such as new information on the suspected action of the drug in the form of additional integrity constraints, such as that the drug cannot induce together a set of molecules or that it can only induce a molecule if some other molecules are not active.

Also the biologist can examine how would the results be affected if we assume that there are some extra reactions (links) in the background network of pathways? For example, the biologists may suspect that there might exist a new inducement link between two molecules. Adding this extra link and reanalyzing the same observed data can give valuable glues as to whether this is plausible and how it would be experimentally confirmed. This process of hypothesizing in a controlled manner new structures on the underlying network and analyzing their significance can be semi-automated. We are developing tools that would assist the biologist in such thought experiments.

Overall Evaluation of ApoCelSys as an Assistant. From a working biologist point of view the overall evaluation of our system is summarized in the following. The ability to input our actual results in an automated assistant that contains all the information needed to describe the cellular microenvironment provides a much simpler alternative than in vitro screening of a large number of proteins. Also the capacity to adjust this microenvironment in order to depict the variability that exists between cell lines greatly enhances the usefulness of this system. The major benefit of this assistant is the frequency distribution charts which provide an indication for which molecules to be investigated next, thus saving time and cutting laboratory costs. Furthermore, the ApoCelSys system may serve as a tool for the discovery of unknown reactions between molecules that can reveal novel pathways to be targeted in cancer therapy.

\subsection{Evaluation of Computational Behaviour}

We have carried out a series of experiments to investigate the computational properties of the current (and first) implementation of the system. This implementation relies on a new implementation of the A-system [5] for computing 
(minimal) explanations but does not in its current form exploit the constraint solving capabilities of the A-system. The response time of the system is dependent on the users' input and may vary from a few seconds to a couple of hours for a complete search of all minimal explanations.

We have thus tried to investigate to what extend it is necessary to compute all explanations and how we can find a representative sample of these. Under the assumption that the effect of the drug to produce the results on the molecules that we have observed in the experiment cannot be "too far" along the pathways from these observed molecules, we have restricted the depth of search by the Asystem and have tried to learn what is a reasonable threshold on the search depth bound. Using the real experiments described above as a basis and running the system with various depth limits we obtain the results shown in figure 5. According to these we can see that no major fluctuation in the nine most probable hypotheses of induced molecules except for a single molecule, bid, on the lowest depth limit of 200. The same can also be observed in figure 6 in which all molecules found in figure 5 have been sorted by their inducement probability and labeled with non-zero positive numbers, where one stands for the most probable molecule to be induced by the substance. In this figure, we can observe that most of the molecules retain their positions, which shows that even in low depth searches, the results provided are of satisfactory quality.

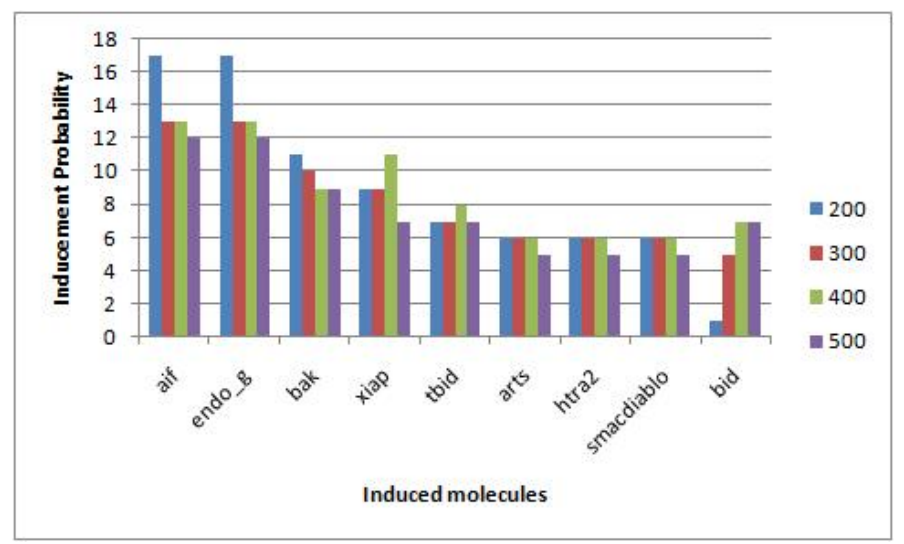

Fig. 5. Search Depth Variation of Explanations

However restricting the search could result in loosing valuable information when our assumption of no "long-distance" effect is not appropriate. The large computational time of an unrestricted search is due to the complex pathways and the fact that the top down search of the A-system contains parts that are unnecessarily duplicated. We plan to develop other implementations that are based on bottom up computational models using tabling or ASP solvers. 


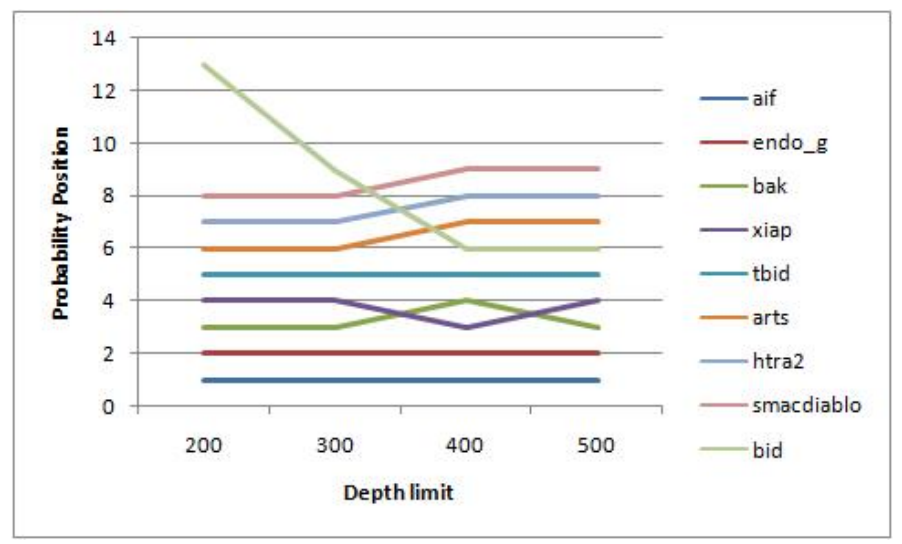

Fig. 6. Probability of drug inducement of molecules

\section{Related Work}

There are several earlier works of Symbolic Systems Biology which also use a form of logical modeling within Logic Programming (Abductive or Inductive) and rely on abductive and/or inductive reasoning for the analysis of experimental results. Some of the earliest such approaches include [15] for the analysis of genetic networks based on abduction, 7] for generating gene interaction networks from micro-array experiments within ALP and 810 for discovering the function of genes from auxotrophic metabolic experiments or for studying lethal synthetic gene mutations using the XHAIL framework and system [9] that supports abductive and inductive reasoning within the representation framework of ALP. In [14] a logical model for capturing the possible toxic effects of drugs in a cell has been developed within ALP and analyzed using the Progol5 system.

Our work can be seen to follow this line of approach, with an emphasis on providing tools with interactive interfaces to help the biologist as an automated scientific assistant. As the underlying network information increases, it is evident that we need to develop a better graphical interface of our system in the form of a map-like interface where the user can focus on the details of the pathways network that $\mathrm{s} /$ he is currently investigating. Similarly, new interactive interfaces are needed to facilitate the thought experimentation process by the user.

More recently, 13. have developed an important methodology for qualitative logical modeling in systems biology that rests on underlying quantitative models for the chemical reactions in the signalling pathways. Our approach lacks this link to quantitative computational models and it would benefit from their integration in the logical model as black computational boxes. This work uses a probabilistic method to rank the multiple hypotheses found much in the same spirit as we do with the frequency charts of individual hypotheses over the set of explanations. They then go a step further to rank the full explanations. This is an extension that would be useful to consider in our approach. 
The authors in 12 show how using the XHAIL system it is possible to allow abductive reasoning for the revision (deletion or addition of reactions) of the underlying pathways on which a logical model for metabolism is build. In our approach and system this is done semi-automatically by suggestions of the expert biologists in the context of possible thought experiments that they want to carry out for further investigation. It would then be useful to automate these type of thought experiments by incorporate the same type of use of abductive reasoning in our model.

Another piece of work that is relevant to our future work is that of [1] where Answer Set Programming is used for the computation of processes of biochemical reaction networks that combines numerical weight information and logical inference. The use of ASP solvers for the computation of abductive reasoning could help improve the computational behavior of our system especially when the network of reaction pathways contains cycles.

\section{Conclusions and Future Work}

We have presented a methodology for building automated scientific assistants to help in the research effort of molecular biologists. This is based on logical modelling of the existing background knowledge of the phenomena studied and the automation of abductive reasoning to provide explanations for the observed empirical results of the experiments carried out by the biologists. The resulting tools could also help in assisting with the further development of the research program of the biologists by facilitating the scientific thinking carried out when decisions for the future direction of research are taken. This methodology and resulting automated assistant, called ApoCelSys, has been tested in a real life case of a research group that studies the chemoprevention of cancer.

The testing of the ApoCelSys system carried out so far provides encouraging results but further systematic experimentation with the system is needed. In particular, it is important to carry out a series of tests to understand better the capabilities of the system for facilitating thought experiments and what extra features and functionality would make it more suitable for this purpose.

\section{References}

1. Papas, A., Constantinou, C., Constantinou, A.: Vitamin e and cancer: An insight into the anticancer activities of vitamin e isomers and analogs. Int. J. Cancer 123(4), 739-752 (2008)

2. Elmore, S.: Apoptosis: a review of programmed cell death. Toxicol Pathol. 35(4), 495-516 (2007)

3. Denecker, M., Kakas, A.C.: Abduction in logic programming. In: Kakas, A.C., Sadri, F. (eds.) Computational Logic: Logic Programming and Beyond. LNCS (LNAI), vol. 2407, pp. 402-436. Springer, Heidelberg (2002)

4. Kakas, A.C., Kowalski, R.A., Toni, F.: Abductive Logic Programming. Journal of Logic and Computation 2(6), 719-770 (1993) 
5. Ma, J., et al.: A-system v2 (2011), http://www-dse.doc.ic.ac.uk/cgi-bin/moin.cgi/abduction

6. Neuzil, J., et al.: Vitamin e analogues as a novel group of mitocans: anti-cancer agents that act by targeting mitochondria. Mol. Aspects Med. 28(5-6), 607-645 (2007)

7. Papatheodorou, I., Kakas, A.C., Sergot, M.J.: Inference of gene relations from microarray data by abduction. In: Baral, C., Greco, G., Leone, N., Terracina, G. (eds.) LPNMR 2005. LNCS (LNAI), vol. 3662, pp. 389-393. Springer, Heidelberg (2005)

8. Ray, O.: Automated abduction in scientific discovery. In: Model-Based Reasoning in Science, Technology, and Medicine, pp. 103-116 (2007)

9. Ray, O.: Nonmonotonic abductive inductive learning. J. Applied Logic 7(3), 329-340 (2009)

10. Ray, O., Bryant, C.H.: Inferring the function of genes from synthetic lethal mutations. In: CISIS, pp. 667-671 (2008)

11. Ray, O., Soh, T., Inoue, K.: Analyzing pathways using ASP-based approaches. In: Horimoto, K., Nakatsui, M., Popov, N. (eds.) ANB 2010. LNCS, vol. 6479, pp. 167-183. Springer, Heidelberg (2012)

12. Ray, O., Whelan, K.E., King, R.D.: Logic-based steady-state analysis and revision of metabolic networks with inhibition. In: CISIS, pp. 661-666 (2010)

13. Synnaeve, G., Inoue, K., Doncescu, A., Nabeshima, H., Kameya, Y., Ishihata, M., Sato, T.: Kinetic models and qualitative abstraction for relational learning in systems biology. Bioinformatics, 47-54 (2011)

14. Tamaddoni-Nezhad, A., Chaleil, R., Kakas, A.C., Muggleton, S.: Application of abductive ilp to learning metabolic network inhibition from temporal data. Machine Learning 64(1-3), 209-230 (2006)

15. Zupan, B., Bratko, I., Demšar, J., RobertBeck, J., Kuspa, A., Shaulsky, G.: Abductive inference of genetic networks. In: Quaglini, S., Barahona, P., Andreassen, S. (eds.) AIME 2001. LNCS (LNAI), vol. 2101, pp. 304-313. Springer, Heidelberg (2001) 